\title{
Connaissances en matière de radioprotection des manipulateurs de radiologie de Bangui et de Bimbo (Centrafrique)
}

\author{
F. Kouandongui Bangue Songrou ${ }^{1, *}$, E. Bidan Tapiade ${ }^{2}$, M. Ouimon ${ }^{3}$ et T. Mobima ${ }^{4}$ \\ ${ }^{1}$ Service de Radiologie et d'Imagerie Médicale du Centre Hospitalier Universitaire Maman Elisabeth DOMITIEN (CHUMED), Bimbo, \\ Centrafrique. \\ ${ }^{2}$ Faculté des Sciences de la Santé de l'Université de Bangui (Centrafrique), Bangui, Centrafrique. \\ ${ }^{3}$ Service de Radiologie et d'Imagerie Médicale de l'Hôpital de l'Amitié (Centrafrique), Bangui, Centrafrique. \\ ${ }^{4}$ Chef de Service de Radiologie et d'Imagerie Médicale de 1'Hôpital de l'Amitié (Centrafrique), Bangui, Centrafrique.
}

Reçu le 20 septembre 2018 / Accepté le 17 décembre 2018

\begin{abstract}
Résumé - Les rayons X, principales sources d'exposition aux rayonnements ionisants (RI) d'origine humaine ont un rôle nocif dans la genèse des affections malignes et cancéreuses. L'application des principes de radioprotection par l'ensemble du personnel utilisant les rayons X est une obligation réglementaire. L'Agence Internationale de l'Energie Atomique (AIEA) recommande que ce personnel soit formé, avec des aptitudes et compétences vérifiées avant d'effectuer les tâches spécifiques. Une enquête a été menée auprès du personnel travaillant sous RI dans les établissements de radiodiagnostic de Bangui et de Bimbo pour évaluer leurs niveaux de connaissances en radioprotection. Parmi les enquêtes, 36,8\% étaient titulaires d'un diplôme de technicien supérieur en radiologie et imagerie médicale, formés à la radioprotection et sans renouvellement de leurs connaissances. Le niveau de connaissance de l'ensemble du personnel, quel que soit le profil et l'établissement est très modeste. L'application du principe de justification des examens n'est pas effective dans tous les établissements. Aucun contrôle qualité n'a jamais eu lieu dans les établissements, ceux-ci manquent de personnel référent en radioprotection et de matériel de protection contre les rayons $\mathrm{X}$. Le gouvernement et l'ANR doivent poursuivre leurs efforts pour la formation continue en radioprotection du personnel et pour le contrôle qualité des installations. La diffusion de la culture de radioprotection entre praticiens est aussi à encourager.
\end{abstract}

Mots clés : radioprotection / radiodiagnostic / manipulateurs / Centrafrique

\begin{abstract}
Knowledge in radiation protection of operators in medical imaging in Bangui and Bimbo (Central Africa Republic). X-rays, main source of exposure to ionizing radiation (IR) of human origin have a harmful role in genesis of malignant and cancerous diseases. The application of the principles of radiation protection by all staff using $\mathrm{x}$-ray is a regulatory obligation. International Atomic Energy Agency (IAEA) recommends that all staff have to be trained with capabilities and competences checked up before performing specific task. A survey was conducted with personnel working under IR in Bangui and Bimbo radiodiagnosis establishments to assess their levels of knowledge in radiation protection. Among the respondents, $36.6 \%$ had a degree of senior technician in radiology and medical imaging, trained in radiation protection without renewal of knowledge. The level of knowledge of all staff, regardless of the profile and the establishment is very modest. Application of principle of justification of exams is not effective in all establishments. Quality control never took place in establishments, they lack anyone to refer in radiation protection and protection against X-ray equipment. Government and ANR must continue to work together for continuing education in radiation protection of the staff and for quality control.
\end{abstract}

Keywords: radiation protection / radio diagnosis / operators / Central African Republic

\footnotetext{
*Auteur de correspondance : fkouando@gmail.com
} 


\section{Introduction}

La radiologie conventionnelle est une technique d'imagerie médicale qui utilise les rayons $X$ pour obtenir une image radiante dans un but diagnostic ou thérapeutique. Depuis la découverte des rayons $X$, leur utilisation en milieu médical ne cesse d'augmenter. Ces RI constituent de très loin la principale source d'exposition aux RI d'origine humaine (Gervaise et al., 2011). L'exposition de la population aux rayonnements ionisants aux États-Unis par an et par individu a été multipliée par six depuis les années quatre-vingts (NCRP, 2009). En France, selon un bilan établi par l'Institut de radioprotection et de sûreté nucléaire (IRSN), la dose efficace moyenne reçue par habitant entre 2007 et 2012 a augmenté près de $20 \%$ (ISRN, 2014). En République Centrafricaine, les données ne sont pas disponibles.

Plusieurs études ont montré le rôle nocif des rayons $\mathrm{X}$ notamment dans la genèse des affections malignes et cancéreuses justifiant le terme de «cancer radio-induit» (Roudier et al., 2006 ; Berrington de Gonzalez et al., 2009). La Commission internationale de protection radiologique (CIPR) recommande la mise en place d'un département de radioprotection chargé de veiller au respect des prescriptions et réglementer l'introduction et l'exercice de toute pratique mettant en jeu des sources de RI, de surveiller les mesures de protection et de sûreté radiologique (ICRP, 1991).

L'application des principes de radioprotection par l'ensemble du personnel directement affectés aux travaux sous rayonnements (DATR) est une obligation réglementaire. Ce personnel est l'un des principaux acteurs dans un programme de radioprotection. L'AIEA dans la collection «Normes de Sûreté » recommande que le personnel utilisant les rayons $\mathrm{X}$ doit être formé, avoir des aptitudes et des compétences à effectuer sans risque les tâches spécifiées et doit être évalué avant toute affectation non supervisée (IAEA, 2001).

Nous avons entrepris ce travail dans le but d'évaluer les connaissances des manipulateurs de radiologie en matière de radioprotection. À notre connaissance, aucune étude portant sur ce thème n'a été réalisée en Centrafrique.

\section{Matériel et méthode}

C'était une étude descriptive réalisée entre juillet et août 2018 sous forme d'enquête auprès des manipulateurs de radiologie des hôpitaux et des cabinets de radiologie de la ville de Bangui et de Bimbo (hôpital de l'Amitié de Bangui (HA), hôpital communautaire de Bangui (HC), complexe pédiatrique de Bangui (CP), centre de radiologie et d'imagerie médicale (CERIM), cabinet médical et d'imagerie diagnostic (CMID), Centre Hospitalier et Universitaire Maman Elisabeth DOMITIEN de Bimbo (CHUMED). Le matériel utilisé était constitué de fiches questionnaires élaborées à partir des données de la littérature (Gervaise et al., 2011; Kouamé et al., 2012). Les manipulateurs de radiologie ayant librement consenti à répondre au questionnaire après une séance d'information ont été retenus pour l'étude.

La première partie du questionnaire était en rapport avec les données démographiques du praticien (l'âge, le sexe, la qualification professionnelle, l'établissement d'appartenance,
Tableau 1. Répartition des personnels interrogés selon leur établissement.

Table 1. Distribution of interviewed staff according to their establishment.

\begin{tabular}{lcc}
\hline Établissements & Effectifs & Pourcentages \\
\hline CHUMED & 5 & 26,3 \\
HC & 5 & 26,3 \\
HA & 4 & 21,1 \\
CP & 2 & 10,5 \\
CERIM & 2 & 10,5 \\
CMCR & 1 & 5,3 \\
Total & 19 & 100 \\
\hline
\end{tabular}

les années d'expérience, la formation spécifique en radioprotection). La deuxième partie avait abordé le niveau de connaissances des principes et des normes de radioprotection par le personnel (la connaissance du principe de justification (1 point), l'application du principe de justification des examens radiographiques (2 points), l'existence des cancers radioinduits ( 1 point), la connaissance des moyens de protection contre les RI (3 points), la prise en compte des dernières dates des règles (1 point) et la prise en compte d'éventuelle grossesse (1 point), faire un test de grossesse en cas de doute (1 point) avant la réalisation des examens. Chaque réponse juste aux questions était notée 01 point, 0 point était affecté à une réponse fausse ou pas de réponse ou « je ne sais pas ». Un score global de connaissance (SGC) noté sur 10 était attribué pour des réponses justes à toutes les questions. Un SGC entre 2 et 4 points était classé moins bon, entre 5 et 6 points, il était classé moyen. À partir de 7 points il était classé bon. La dernière partie était consacrée à la conformité des installations et des équipements et la présence d'un personnel référent en radioprotection au sein de l'établissement. Le questionnaire avait été remis en mains propres aux manipulateurs des différents établissements de radiologie durant la période du $1^{\text {er }}$ au 10 juillet et, a été récupéré avant le 25 juillet.

\section{Résultats}

\subsection{Profil du personnel}

Dix-neuf personnels DATR sur vingt et un (19/21), répartis dans six établissements de radiologie avaient répondu au questionnaire. Ils étaient tous de sexe masculin, d'âge moyen de 44,58 ans avec les extrêmes de 31 et 55 ans. La répartition des praticiens par établissement figure dans le tableau 1. La distribution du personnel par profil a révélé que $36,8 \%$ des enquêtés étaient titulaires d'un diplôme de technicien supérieur en radiologie et imagerie médicale et $63,2 \%$ du reste du personnel était constitué des infirmiers diplômés d'État (IDE) à hauteur de $26,4 \%$ et des assistants de santé (AS) soit 36,8\%; (Tab. 2). Douze sur dix-neuf (12/19) avaient une expérience professionnelle inférieure à 10 ans sous rayonnements ionisants (RI), $5 / 19$ et $2 / 19$ avaient respectivement travaillé entre 10 et 19 ans et plus de 20 ans sous RI (Tab. 3). 
Tableau 2. Répartition des praticiens selon leur qualification professionnelle.

Table 2. Distribution of the staff according to their vocational qualification.

\begin{tabular}{lcl}
\hline Profils & Effectifs & Pourcentages \\
\hline Techniciens supérieurs en radiologie & 7 & 36,8 \\
Assistants de santé & 7 & 36,8 \\
Infirmiers diplômés d'État & 5 & 26,4 \\
Total & 19 & 100 \\
\hline
\end{tabular}

Tableau 3. Classification des moyennes des scores globaux de connaissance par années d'expérience.

Table 3. Average of global score of knowledge classification according to the years of experience.

\begin{tabular}{llll}
\hline Tranches d'années & Effectifs & SGC extrêmes & Moyennes des SGC \\
\hline $0-09$ ans & 12 & {$[1,6]$} & 3,6 \\
$10-19$ ans & 5 & {$[4,5]$} & 4,6 \\
20 ans et plus & 2 & {$[5,6]$} & 5,5 \\
\hline
\end{tabular}

Tableau 4. Répartition des praticiens selon le score global de connaissance.

Table 4. Distribution of the staff according to the global score of knowledge.

\begin{tabular}{lcl}
\hline SGC & Effectifs & Pourcentages \\
\hline Bon & 0 & 0 \\
Assez bien & 10 & 52,6 \\
Moins bon & 9 & 47,4 \\
Total & 19 & 100 \\
\hline
\end{tabular}

\subsection{Formation en radioprotection}

Tous les techniciens en radiologie et imagerie médicale, soit $36,8 \%$ avaient affirmé avoir reçu un enseignement spécifique en radioprotection durant leur formation initiale mais avaient affirmé ne pas avoir bénéficié d'une formation continue ou d'un recyclage en radioprotection pendant leurs années d'expérience sous rayonnements ionisants. Le reste du personnel, soit $63,2 \%$ avait répondu par la négative en ce qui concerne toute formation en radioprotection.

\subsection{Connaissances des principes et normes de radioprotection}

Les tableaux 4, 5 et 6 résument le niveau de connaissance des principes et normes de radioprotection des personnels DATR. La moyenne du score global de connaissance en radioprotection de tous les praticiens était de $4,05 / 10$, ce qui est classé moins bon; $52,6 \%$ du personnel avait obtenu un SGC assez bien contre $47,3 \%$ de SGC classé moins bon. L'appartenance à un établissement n'était pas associée à une
Tableau 5. Distribution des moyennes de score global de connaissance par établissement.

Table 5. Distribution of averages global score of knowledge according to the establishment.

\begin{tabular}{llll}
\hline Etablissements & Effectifs & SGC extrêmes & Moyennes des SGC \\
\hline CHUMED & 5 & {$[2,6]$} & 3,8 \\
HC & 5 & {$[2,6]$} & 4,0 \\
HA & 4 & {$[1,6]$} & 4,5 \\
CP & 2 & {$[2,5]$} & 3,5 \\
CERIM & 2 & {$[4,5]$} & 4,5 \\
CMID & 1 & & 4,0 \\
\hline
\end{tabular}

Tableau 6. Distribution des moyennes du score global de connaissance par profil.

Table 6. Distribution of averages global score of knowledge according to the vocational qualification.

\begin{tabular}{llll}
\hline Profils & Effectifs & $\begin{array}{l}\text { SGC } \\
\text { extrêmes }\end{array}$ & $\begin{array}{l}\text { Moyenne } \\
\text { du SGC }\end{array}$ \\
\hline Techniciens supérieurs & 7 & {$[5,6]$} & 5,6 \\
Assistants de santé & 7 & {$[1,3]$} & 2,1 \\
Infirmiers diplômés d'État & 5 & {$[4,5]$} & 4,6 \\
\hline
\end{tabular}

meilleure connaissance en radioprotection. Tous les établissements avaient obtenu un SGC en dessous de la moyenne. La moyenne de SGC par établissement était classée moins bon. La connaissance en radioprotection croît avec le nombre d'années d'expérience et le profil du praticien (niveau de connaissance en techniques radiographiques). L'existence du risque de cancer radio-induit a été rapportée par la majorité de nos enquêtés (17/19) soit $89,5 \%$ du personnel interrogé. Le matériel plombé est reconnu par l'ensemble des personnes DATR interrogées comme moyen de protection contre les RI, la distance n'était pas citée comme un autre moyen de protection contre les rayons X par $76,2 \%$ des praticiens (16/ 21). Le principe de justification des examens était reconnu par la majorité des praticiens, soit 85,7\% (18/21), mais n'était pas appliqué par tous. Les prescriptions radiologiques étaient pour la plus part du temps systématiquement réalisées sans remise en question du bien-fondé de la procédure radiologique proposée de peur d'entrer en conflit avec les prescripteurs. Certains examens étaient réalisés uniquement à la demande des patients. L'ensemble du personnel enquêté n'interrogeait jamais les femmes en âge de procréer sur leur date des dernières règles ou sur l'existence d'une éventuelle grossesse.

\subsection{Existence d'une signalisation lumineuse à l'entrée des salles}

Des six services de radiologie enquêtés, seul le service de radiologie du Centre Hospitalier et Universitaire Maman Elisabeth DOITIEN (CHUMED) avait une signalisation lumineuse à l'entrée des salles de radiologie conventionnelle. 


\subsection{Port de dosimètre}

Tous ont répondu par la négative en ce qui concerne le port d'un dosimètre individuel. En dehors des techniciens de radiologie et d'imagerie médicale, tous affirmaient n'avoir jamais vu un dosimètre individuel et ne connaissaient pas son utilité.

\subsection{Contrôle qualité du matériel selon le personnel}

Toutes les réponses étaient négatives en ce qui concerne l'existence d'un contrôle qualité des installations radiologiques de leur service depuis leurs années d'expérience sous RI.

\subsection{Personnel qualifié compétent référent de radioprotection}

Tous les enquêtés avaient répondu qu'il n'existait aucun personnel qualifié compétent référent en radioprotection dans leur établissement.

\section{Discussion}

\subsection{Le profil du personnel}

L'état de la radioprotection dans les pays au sud du Sahara n'est pas optimal (Smith-Bindman et al., 2009) celui de la Centrafrique est très préoccupant au regard de nos résultats. Environ 36,8\% des personnels travaillant sous RI sont des manipulateurs de radiologie et d'imagerie médicale qui ont reçu une formation spécifique en radioprotection durant leur formation initiale. Plus de la moitié du personnel $(63,2 \%)$ n'a jamais été initiée à la radioprotection avant de travailler dans un service de radiodiagnostic. Dans les hôpitaux de Segma de la région Marrakech au Maroc (Jaouad, 2013), 74,3\% des techniciens sont des manipulateurs d'électro radiologie formés à la radioprotection, ce qui contraste avec nos résultats. L'absence d'une école de formation des techniciens en radiologie et imagerie médicale en Centrafrique explique en partie cette faible proportion de personnels qualifiés à la manipulation des RI. L'affectation du personnel sans connaissance en radioprotection dans un service utilisant les RI est contraire aux recommandations de l'AIEA (IAEA, 2001) qui exigent de toute personne travaillant sous les RI à des fins médicales une formation continue en radioprotection.

\subsection{Connaissance des personnels en radioprotection}

Au cours de cette étude, la qualification professionnelle et l'ancienneté dans un service de radiologie sont des facteurs associés à une connaissance des principes et des normes de radioprotection. De façon classique cette constatation est conforme aux travaux des autres auteurs (Ongolo-Zogo et al., 2013) et renforce une fois de plus les recommandations de l'AIEA. Il est donc impératif de mettre en place un programme de formation du personnel avant son affectation dans un service utilisant les rayonnements ionisants. Cette étude a aussi révélé l'absence de renouvellement de connaissance en radioprotection ce qui peut expliquer en partie cette constatation. Le principe de justification, qui est une analyse du rapport bénéfices/risques lié à l'exposition médicale aux RI a été reconnu par l'ensemble du personnel, cependant son application a posé problème. L'application du principe de justification devrait être effective si les examens de radiographie prescrits étaient d'abord validés par le radiologue avant leur réalisation. En réalité, la responsabilité de la justification d'un examen irradiant est partagée entre le radiologue et le prescripteur. Avant la réalisation d'un examen radiologique, le radiologue doit s'assurer de sa justification pour le patient concerné. En cas de désaccord entre le praticien demandeur et le radiologue, la décision de réalisation de l'acte appartient à ce dernier (art. $\mathrm{R}$ 1335-57 du code de santé publique) (Etard, 2010). Le prescripteur devient ainsi un élément important dans le processus de mise en œuvre de la radioprotection et doit à ce titre être formé et doit renouveler ses connaissances tous les dix ans (Nikiema et al., 2017; Nombo et al., 2017). Cette constatation amène certains auteurs (Gervaise et al., 2011) à proposer l'introduction d'un cours sur la radioprotection dans le programme d'études de base des facultés de médecine et d'art dentaire. Toutefois l'amélioration du respect des normes de radioprotection devrait impliquer tout le personnel au contact du patient.

L'absence de dosimètre individuel, l'absence de signalisation à l'entrée des salles de radiologie, l'absence d'un personnel référent en matière de radioprotection et l'absence de contrôle qualité des équipements radiographique sont des défaillances dues à une absence d'un organisme de contrôle et à une absence de culture de sûreté. Cette situation n'est pas propre à la Centrafrique, elle est commune à la plus part des pays au sud du Sahara (Mbo et al., 2017; Nikiema et al., 2017). Elle expose les patients, le personnel médical aux effets néfastes des RI. La dosimétrie individuelle est l'un des maillons essentiels du dispositif de radioprotection des travailleurs exposés aux RI (Rannou, 2006). Elle fournit une estimation des doses reçues au niveau de l'organisme et permet de mettre en œuvre le principe d'optimisation selon lequel les expositions doivent être maintenues au niveau le plus faible qu'il est raisonnablement possible d'atteindre. Une Agence Nationale de Radioprotection de Centrafrique (ANR) a été créée par la loi $\mathrm{n}^{\circ} 06.031$ du 27 septembre 2006 (République centrafricaine, 2006) et dont la mission est d'assurer la réglementation relative à la radioprotection pour toute activité mettant en œuvre des substances radioactives et des sources de RI (République centrafricaine, 2008). Cette agence est déjà dotée d'un cadre juridique et réglementaire pour la protection et la sûreté dans toutes les situations d'exposition (loi 08.011 du 13 février 2008 portant organisation $\mathrm{du}$ cadre institutionnel et juridique applicable aux entreprises et offices publiques) (République centrafricaine, 2008). Pour satisfaire aux obligations internationales le gouvernement devrait veiller à ce que la législation soit explicite sur la surveillance de l'exposition externe du personnel travaillant sous RI, la surveillance de l'environnement, la vérification de la conformité aux normes de sécurité radiologique des installations publiques et privées. Il doit ensuite veiller à ce que les dispositions prévues par les textes soient mises en pratique. 


\section{Conclusion}

Les résultats de cette étude interpellent l'ANR et le Ministère de la Santé Publique sur la nécessité de poursuivre les efforts en formation continue en radioprotection et pour le contrôle des installations. Chaque établissement devrait encourager les praticiens nouvellement formés à diffuser la culture de radioprotection parmi leurs collègues.

\section{Références}

Berrington de Gonzalez A et al. 2009. Projected cancer risks from computed tomography scans performed in the United States in 2007. Arch. Intern. Med. 169(22): 2071-2077.

Etard C. 2010 Les obligations du radiologue pour la radioprotection du patient. J. Radiol. 91: 1207-1211.

Gervaise A, Esperabe-Vignau F, Pernin M, Naulet P, Portron Y, Lapierre-Combes M. 2011. Évaluation des connaissances des prescripteurs de scanner en matière de radioprotection des patients. J. Radiol. 92: 681-687.

IAEA. 2001. Radiological protection for medical exposure to ionizing radiation. Safety standards series $n^{\circ}$ rs-g-1.5. Vienna: IAEA.

ICRP. 1991. ICRP publication 60 (1991): 1990 recommendations of International Commission on Radiologic Protection. Annals of the ICRP. New York, NY: Pergamon Press, p. 68.

ISRN. 2014. Exposition de la population française aux rayonnements ionisants liée aux actes de diagnostic médical en 2012. Fontenayaux-Roses: Institut de Radioprotection de sûreté nucléaire. Rapport PRP-HOM n² 2014-2016.

Jaouad S. 2013. Étude de l'observance des règles de la radioprotection en radiologie conventionnelle dans les hôpitaux Segma de la région Marrakech Tensift al haouz. Maroc: IFCS Rabat. 37 p.

Kouamé N. Ngoan-Domoua AM, Sétchéou A, Nezou BJP, Konan $\mathrm{KD}$, N'gbesso RD, Kéita AK. 2012. Grossesse et risques d'irradiations en radiodiagnostic: État des connaissances des usagers au CHU de Yopougon (Abidjan-Cote d'Ivoire). J. Afr. Imag. Méd. 4(1): 12-20.
Mbo Amvene J, Djonyang B, Mballa Amougou JC, Ngaroua D, Nko'o Amvene S. 2017. Observance des règles de radioprotection dans les services d'imagerie de l'Extrême-Nord du Cameroun. Health Sci. Dis. 18(2): 83-87.

Nikiema Z, Tankoano AI, Bicaba D, Sombie I, Zoungrana B, Cisse R. 2017. Évaluation des connaissances des praticiens sur la radioprotection des patients au Burkina Faso. J. Afr. Imag. Méd. 9(3): 101-105.

Nombo M, Gagna G, Lahutte M, Bourguignon M, Amabile JC. 2017. Évaluation des connaissances acquises par des praticiens des armées à la suite d'une formation réglementaire à la radioprotection des patients. Méd. Armées 45(4): 451-458.

Ongolo-Zogo P, Nguehouo MB, Yomi J, Nko'o Amven S. 2013. Connaissances en matière de radioprotection : enquête auprès des personnels des services hôspitaliers de radiodiagnostic, radiothérapie et médecine nucléaire à Yaoundé Cameroun. Radioprotection 48(1): 39-49.

Rannou A. (2006) Adéquation de la dosimétrie au poste de travail : cas de la dosimétrie individuelle externe. Radioprotection 41(1): 51-63.

République Centrafricaine. 2006. Loi n 06.031 du 27 septembre 2006 sur les minerais radioactifs de la République Centrafricaine.

République Centrafricaine. 2008. Décret $n^{\circ} 08.296$ du 20 août 2008 fixant les modalités d'application de la loi et les présents statuts.

République Centrafricaine. 2008. Loi n 08.011 du 13 février 2008 , portant organisation du cadre institutionnel et juridique applicable aux entreprises et offices publics.

Roudier C, Pirard P, Donadieu J. 2006. Indice des radiodermites secondaires à un geste de radiologie interventionnelle. Presse Méd. 35: 955-959.

Schauer DA, Linton OW. 2009. NCRP Report $\mathrm{N}^{\mathrm{o}} 160$, Ionizing radiation exposure of the population of the United States, medical exposure. Are we doing less with more, and is there a role health physicists? Health Phys. 97(1): 1-5.

Smith-Bindman R, Lisson J, Marcus R, Kim KP, Maheh M, Could R. 2009. Radiation dose associated with common computed tomography examination and the associated life time attributable risk of cancer. Arch. Inter. Med. 169: 2078-2086.

Citation de l'article : Kouandongui Bangue Songrou F, Bidan Tapiade E, Ouimon M, Mobima T. 2019. Connaissances en matière de radioprotection des manipulateurs de radiologie de Bangui et de Bimbo (Centrafrique). Radioprotection 54(1): 41-45 